\title{
Ferritin-Based Nanodrug for the Treatment of Breast Cancer
}

\author{
S. Mazzucchelli \\ Department of Biomedical and Clinical Sciences "L. Sacco" \\ University of Milan \\ Milan, Italy \\ Serena.Mazzucchelli@unimi.it
}

Breast Cancer (BC) is the most frequently diagnosed malignancy and the second leading cause of cancer-related death among women. Nowadays two distinct BC subtypes represent a critical clinical challenge: 1) HER2+ BC and 2) Triple Negative BC (TNBC). The former is currently treated by targeted anti-HER 2 antibodies, but it is characterized by the frequent onset of resistance leading to disease recurrence. The TNBC displays heterogeneity, immunogenicity and poor prognosis and no targeted therapy is currently available. Hence, both $\mathrm{BC}$ subtypes are treated with cytotoxic chemotherapy combined (HER2+-BC) or not (TNBC) with other targeted drugs [1].

Main achievements in breast cancer treatment would be obtained by the generation of smart drug delivery systems able to allow a targeted release of chemotherapeutics in cancer cells. In this scenario, protein-based nanocages hold a tremendous potential as devices for targeted nano-drug delivery [2].

In particular, apoferritin nanocages has emerged as an excellent and promising protein-based device thanks to its unique architecture, surface properties and high biocompatibility [3, 4]. By exploiting natural recognition of the Transferrin Receptor 1 , which is overexpressed on tumor cells [5], apoferritin nanocages may ensure a proper drug delivery and release [6], and also the availability to a whole body treatment, thanks to its capability to cross the biological barriers [7]. Moreover, researchers have applied surface functionalities on ferritin cages for further providing active tumor targeting [8]. Encapsulation strategies of non metal-containing drugs within ferritin cages have been explored and successfully performed with encouraging results [9, 10]. Various preclinical studies have demonstrated that nanoformulation within ferritin nanocages significantly improved the therapeutic index of encapsulated drugs, thanks to targeted treatment of cancer cell and a reduced activity in off-target organs or tissues $[11,12]$. Here, we report all the nanotechnological approaches implemented for applying them to cancer treatment and the promising results obtained.

\section{References}

[1] R. L. N. Godone, G. M. Leitão, N. B. Araújo, C. H. M. Castelletti, J. L. Lima-Filho JL, D. B. G. Martins, "Clinical and molecular aspects of breast cancer: Targets and therapies," Biomed Pharmacother., vol. 106, pp. 14, 2018.

[2] M. Colombo, F. Corsi, D. Foschi, E. Mazzantini, S. Mazzucchelli, C. Morasso, E. Occhipinti, L. Polito, D. Prosperi, S. Ronchi, P. Verderio, "HER2 targeting as a two-sided strategy for breast cancer diagnosis and treatment: Outlook and recent implications in nanomedical approaches," Pharmacol Res., vol. 62, no. 2, pp. 150, 2010.

[3] M. Truffi, L. Fiandra, L. Sorrentino, M. Monieri, F. Corsi, S. Mazzucchelli, "Ferritin nanocages: A biological platform for drug delivery, imaging and theranostics in cancer," Pharmacol. Res., vol. 107, pp. 57, 2016.

[4] F. Corsi, S. Mazzucchelli, "The potential of protein-based nanocages for imaging and drug delivery," Ther Deliv., vol. 7, no. 3, pp.149, 2016.

[5] K. Fan, C. Cao, Y. Pan, D. Lu, D. Yang, J. Feng, L. Son, M. Liang, X. Yan, "Magnetoferritin nanoparticles for targeting and visualizing tumour tissues," Nat Nanotechnol., vol. 7, no.7, pp. 459, 2012.

[6] M. Bellini, S. Mazzucchelli, E. Galbiati, S. Sommaruga, L. Fiandra, M. Truffi, M. A. Rizzuto, M. Colombo, P. Tortora, F. Corsi, D. Prosperi, "Protein nanocages for self-triggered nuclear delivery of DNA-targeted chemotherapeutics in Cancer Cells," J Control Release., vol. 196, pp. 184-96, 2014.

[7] L. Fiandra, S. Mazzucchelli, M. Truffi, M. Bellini, L. Sorrentino, F. Corsi, "In Vitro Permeation of FITC-loaded Ferritins Across a Rat Blood-brain Barrier: a Model to Study the Delivery of Nanoformulated Molecules," J Vis Exp., vol. 114, 2016. 
[8] Z. Zhen, W. Tang, H. Chen, X. Lin, T. Todd, G. Wang, T. Cowger, X. Chen, J. Xie, "RGD-modified apoferritin nanoparticles for efficient drug delivery to tumors," ACS Nano., vol. 7, no. 6, pp. 4830-7, 2013.

[9] S. Mazzucchelli, M. Truffi, F. Baccarini, M. Beretta, L. Sorrentino, M. Bellini, M. A. Rizzuto, R. Ottria, A. Ravelli, P. Ciuffreda, D. Prosperi, F. Corsi, "H-Ferritin-nanocaged olaparib: a promising choice for both BRCA-mutated and sporadic triple negative breast cancer," Sci Rep., vol. 7, no. 1, pp. 7505, 2017.

[10] L. Pandolfi, M. Bellini, R. Vanna, C. Morasso, A. Zago, S. Carcano, S. Avvakumova, J. A. Bertolini, M. A. Rizzuto, M. Colombo, D. Prosperi, "H-Ferritin Enriches the Curcumin Uptake and Improves the Therapeutic Efficacy in Triple Negative Breast Cancer Cells," Biomacromolecules., vol. 18, no. 10, pp. 3318, 2017.

[11] L. Zhang, L. Li, A. Di Penta, U. Carmona, F. Yang, R. Schöps, M. Brandsch, J. L. Zugaza, M. Knez, "H-Chain Ferritin: A Natural Nuclei Targeting and Bioactive Delivery Nanovector," Adv Healthc Mater., vol. 4, no. 9, pp. 1305-10, 2015.

[12] S. Mazzucchelli, M. Bellini, L. Fiandra, M. Truffi, M. A. Rizzuto, L. Sorrentino, E. Longhi, M. Nebuloni, D. Prosperi, F. Corsi, "Nanometronomic treatment of 4T1 breast cancer with nanocaged doxorubicin prevents drug resistance and circumvents cardiotoxicity," Oncotarget., vol. 8, pp. 8383, 2017. 